\title{
Iris Code Capacity Analysis
}

This paper was downloaded from TechRxiv (https://www.techrxiv.org).

\section{LICENSE}

CC BY-NC-SA 4.0

SUBMISSION DATE / POSTED DATE

07-08-2021 / 11-08-2021

CITATION

Zuo, Jinyu; Schmid, Natalia (2021): Iris Code Capacity Analysis. TechRxiv. Preprint. https://doi.org/10.36227/techrxiv.15128643.v1

$\mathrm{DOI}$

10.36227/techrxiv.15128643.v1 


\section{Iris Code Capacity Analysis}

\author{
$1^{\text {st }}$ Jinyu Zuo \\ Lane Department of CSEE \\ West Virginia University \\ Morgantown, WV, USA \\ jzuo@mix.wvu.edu
}

\author{
$2^{\text {nd }}$ Natalia A. Schmid \\ Lane Department of CSEE \\ West Virginia University \\ Morgantown, WV, USA \\ Natalia.Schmid@mail.wvu.edu
}

\begin{abstract}
Daugman's design of IrisCode continues fascinating the research world with its practicality, efficiency, and outstanding performance. The limits of Daugman's recognition system, however, remain unquantified. Multiple approaches to scale performance have been explored in the past. Despite them, the problem of finding the capacity of IrisCode remains open.

In an attempt to fill the gap in understanding the performance limits of Daugman's algorithm, we turn to an analysis of the relationship between the size of the population that the IrisCode can effectively cover and the iris sample quality. Given Daugman's IrisCode algorithm, the problem of finding its capacity is cast as a basic Rate-Distortion/Channel Coding problem. The Hamming, Plotkin, and Elias-Bassalygo upper bounds on the population of a binary code under the constraint of a minimum Hamming Distance between any two codewords is applied to relate the number of iris classes that the IrisCode algorithm can sustain and the quality of iris data expressed in terms of Hamming Distance.
\end{abstract}

Index Terms-IrisCode, capacity, iris image quality, RateDistortion, Hamming, Plotkin, and Elias-Bassalygo bounds

\section{INTRODUCTION}

As any practical data, iris data are not perfect. Even good quality iris images experience some quality degradation due to occlusion, illumination condition, camera noise, motion, and out-of-focus blurs, followed by an additional degradation due to imperfect signal processing applied while iris images are transformed to IrisCode templates. All these degradations make perfect zero genuine scores impossible. When partitioning the interval of normalized Hamming distances (HDs) into genuine and imposter subintervals, 0.32 is traditionally used as the threshold. This implies that two IrisCode templates are considered as samples from the same class if the normalized HD between them is less than 0.32 .

Without rotation (head tilt) compensation, the false positive rate in a verification scenario can be calculated by fitting a Binomial distribution with a degree of freedom equal to the number of independent bits. Consider Rank 1 recognition scenario. If the number of degrees of freedom is set to 245 as claimed by Daugman [1] (249 as claimed in an earlier paper [2]), then the false positive rate at 0.32 threshold is equal to $0.7292 \times 10^{-8}$. It means that a sample from a new class (not present in the iris dataset), will be classified as one of the existing classes with a chance one in $1.3713 \times 10^{8}$. At first, it looks impressive. However, if an iris system has already one billion classes in it, then the chance that the sample from a new class is classified as at least one of the existing classes is

$$
1-\left(1-0.7292 \times 10^{-8}\right)^{10^{9}}=99.93 \% \text {. }
$$

This means that the population of $10^{9}$ (or $0.5 \times 10^{9}$, if we consider two eyes per person) may already exceed the value of capacity of the Daugman's system, because for any iris class there may be at least one more class in the database that is very similar to it.

Daugman's model for the rate of false positives relies on the number of independent bits, which is an indicator of the strength of IrisCode. This model shows excellent outcomes when applied in a verification setting, where every imposter match assumes a comparison of two randomly selected IrisCode templates. In this paper, we propose an approach to find the maximum possible population of iris classes that IrisCode accommodates. The approach appeals to Rate-Distortion/Channel Coding theory. It takes into account the distribution of iris data within and across iris classes and also of the quality of iris data. Putting it in terms of biometric terminology, the proposed approach is suitable to evaluate the population limits of a closed iris recognition system performing Rank 1 recognition.

The rest of the paper is organized as follows. Section II presents three bounds on the maximum population that IrisCode can sustain for the case when enrolled iris classes are characterized by nearly perfect quality. Section III describes the situation when the quality of iris data is not so perfect. Finally, Section IV provides a summary of the main points discussed in the paper.

\section{NOISE FREE ENROLLMENT}

In the rest of the paper, we assume that a one-to-one encoding technique is available to map Daugman's IrisCode templates (binary codewords representing iris classes) into a set of binary codewords, each of length $n=245$ independent bits. Note that the length of each codeword is equal to the number of degrees of freedom supported by IrisCode. Given this assumption, the problem of finding the maximum population covered by Daugman's algorithm is reduced to finding the number of binary codewords with a specified minimum HD between any two codewords as a constraint.

Let us assume for a moment that all enrolled iris classes are represented by noise-free iris codewords and attribute 
distortions and noise that reflect on the value of normalized HD to a query iris image submitted for recognition. Consider binary codewords of length $n$ and denote the threshold that separates the interval of possible normalized HDs into genuine and imposter subintervals as $\epsilon$. Given the assumption above, ideal iris classes can be visualized as points positioned along the $n$-dimensional lattice in a $2^{n}$ code space. If a query iris codeword, submitted for recognition, belongs to a specific iris class, then the query codeword and the true codeword of the claimed class lie within a hyper-dimensional ball of radius $r$, where $r$ and $\epsilon$ are related as

$$
r=\lfloor\epsilon \times n\rfloor \text {, bits. }
$$

For iris classes to be distinguishable during matching, centers of the hyper-dimensional balls representing different iris classes have to be spaced at least $r \times 2+1=\lfloor\epsilon \times n\rfloor \times 2+1$ bits apart. Under this setup, the problem of finding the capacity of IrisCode is reduced to the sphere packing problem from Rate-Distortion/Channel Coding theory [3]. This problem has been well analyzed, and the developed results can be directly applied to estimate the size of the IrisCode population. Most sphere packing results are presented in the form of bounds. Below we provide a brief summary of three bounds and illustrate how to apply them to IrisCode.

\section{A. Hamming Bound}

Let $C(n, r)$ denote the maximum possible population of a binary iris gallery with each iris class represented by a codeword of length $n$, given that the minimum HD between iris classes equals $2 \times r+1=\lfloor\epsilon \times n\rfloor \times 2+1$ bits. Then, the application of the Hamming bound [4] yields the following result

$$
C(n, r) \leq \frac{2^{n}}{\sum_{i=0}^{r}\left(\begin{array}{l}
n \\
i
\end{array}\right)}
$$

For illustration, we set $n$ to 245 . Then the number of codewords in unconstrained code is equal to $2^{245}$. If the decision threshold $\epsilon$ is set to 0.32 , the maximum radius of a class hyper-dimensional ball $r$ is equal to

$$
r=\lfloor 245 \times 0.32\rfloor=\lfloor 78.40\rfloor=78, \text { bits. }
$$

Then the minimum HD between iris codewords is $2 \times r+1=$ 157 bits and the Hamming bound on the number of possible iris classes yields

$$
C(245,78) \leq\left\lfloor 2^{245} / \sum_{i=0}^{78}\left(\begin{array}{c}
245 \\
i
\end{array}\right)\right\rfloor=151.61 \times 10^{6} .
$$

Table I presents the results of Hamming Bound as a function of normalized HD.

\section{B. Plotkin Bound}

The Hamming bound is considered to be a loose upper bound on a constrained code population. Among other bounds, the Plotkin bound [5] and the Elias-Bassalygo bound are tighter alternatives, although each has its own limitations.
Under condition $(4 r+3)>n$, the Plotkin bound on the population covered by IrisCode is given as

$$
C(n, r) \leq 2\left\lfloor\frac{2 r+2}{4 r+3-n}\right\rfloor \text {. }
$$

If $(4 r+3)=n$, then the Plotkin bound can be calculated as

$$
C(n, r)=C(4 r+3, r) \leq 8 r+8 .
$$

To illustrate performance of the bound, we continue the example from the previous subsection. We set $n=245$ and $\epsilon=0.32$. Thus, the radius of the hyper-dimensional ball is $r=78$ bits. The Plotkin upper bound on the number of total possible iris classes yields

$$
C(245,78) \leq 2\left\lfloor\frac{158}{70}\right\rfloor=4 .
$$

Table II displays the results of the Plotkin bound as a function of two parameters, codeword length $n$ and threshold $\epsilon$. As can be seen from the table, for a large value of normalized HD (the same as threshold $\epsilon$ ), the population coverage of IrisCode is limited to only a few classes and the number of classes does not change much even when the length of codewords is doubled.

\section{Elias-Bassalygo Bound}

Both Hamming and Plotkin bounds are not tight when the threshold $\epsilon$ is set to a small value. As an alternative solution, we involve Elias-Bassalygo bound [6]. This bound is known to be tight for small values of threshold $\epsilon$ and large values of $n$.

Under condition $(2 r+1) \leq n / 2$, the Elias-Bassalygo bound on the population covered by IrisCode is given as

$$
C(n, r) \leq\left\lfloor\frac{n 2^{n+1}}{\left(\begin{array}{c}
n \\
J(n, r)
\end{array}\right)}\right\rfloor
$$

where

$$
J(n, r)=\left\lfloor\frac{n}{2}\left(1-\sqrt{1-\frac{2(2 r+1)}{n}}\right)\right\rfloor .
$$

A comparison of the three bounds for code length 245 and varying values of threshold $\epsilon$ is presented in Fig. 1. Numerical values are provided in Table III.

\section{Comments on Performance}

A numerical comparison between the Hamming bound, Plotkin bound, and empirically evaluated capacity is provided in Table $\mathrm{V}$ for $n=16$, where the empirical capacity is obtained through an exhaustive search. The search is implemented in several steps. It begins with generating all possible binary codewords of length $n$. Then a single codeword is picked and all its neighbor codewords with the normalized HD smaller than $\epsilon$ are eliminated. This step is followed by analyzing the remaining neighbor codewords and eliminating their neighbors that are located closer than what is allowed by 
TABLE I

HAMMING BOUND FOR CODE LENGTH $n=245$

\begin{tabular}{|c||c|c|c|c|c|}
\hline \multicolumn{1}{|c||}{} & \multicolumn{5}{c|}{ Radius in Normalized Hamming Distance } \\
\cline { 2 - 6 } & 0.28 & 0.30 & 0.32 & 0.34 & 0.36 \\
\hline Radius $r$ in bits & 68 & 73 & 78 & 83 & 88 \\
\hline Minimum HD in bits & 137 & 147 & 157 & 167 & 177 \\
\hline Hamming Bound & $8.87 \times 10^{11}$ & $9.14 \times 10^{9}$ & $1.52 \times 10^{8}$ & $3.98 \times 10^{6}$ & $1.62 \times 10^{5}$ \\
\hline
\end{tabular}

TABLE II

PLOTKIN BOUND FOR CODE LENGTHS $n=256,512,1024$, AND 2048

\begin{tabular}{|c||c|c|c|c|c|c|}
\hline \multicolumn{1}{|c||}{} & \multicolumn{5}{c|}{ Radius in Normalized Hamming Distance } \\
\cline { 2 - 7 } & 0.24 & $\mathbf{0 . 2 5}$ & $\mathbf{0 . 2 6}$ & $\mathbf{0 . 2 7}$ & 0.28 & 0.32 \\
\hline$n=256$ & & 86 & 24 & 12 & 8 & 4 \\
\hline$n=512$ & & 172 & 22 & 12 & 8 & 4 \\
\hline$n=1024$ & & 342 & 24 & 12 & 8 & 4 \\
\hline$n=2048$ & & 684 & 24 & 12 & 8 & 4 \\
\hline
\end{tabular}

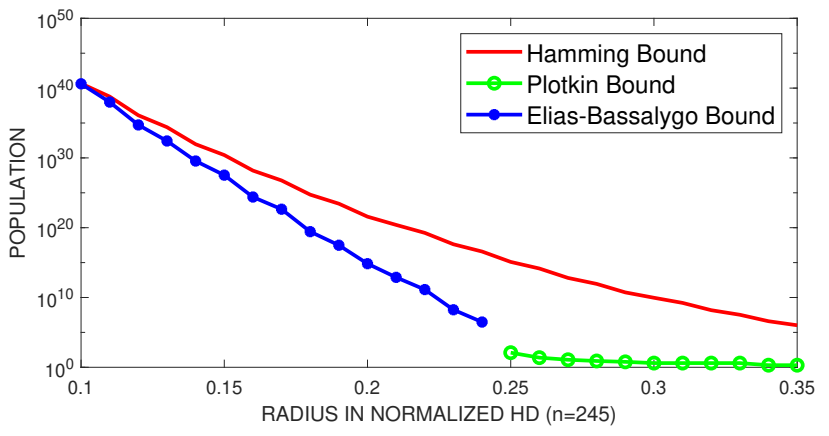

Fig. 1. Hamming, Plotkin, and Elias-Bassalygo bounds for code length 245

the normalized HD. This process continues until all pairs of codewords are spaced at least $\lfloor\epsilon \times n\rfloor \times 2+1$ apart.

Among the three bounds, the Elias-Bassalygo bound performs poorly when $n$ is small. These numbers were not included in the table. Also, from the table, it is interesting to note that bounds can be quite loose. In some cases, bounds can take values $300 \%$ larger than the empirically evaluated population limit.

\section{E. Effect of the Head Tilt}

Until now the effect of head tilt, stated and addressed as the problem of the best alignment of two IrisCode templates, has not been addressed. The code alignment by shifting IrisCode templates right and left by several degrees will affect the value of IrisCode capacity. A numerical comparison of the IrisCode population with and without code alignment ( \pm 1 bit rotation alignment) is provided in Table VI. The population values were obtained by applying an exhaustive search. From the analysis of the values, we note that for small values of the radius of class hyper-dimensional balls, the maximum population of the system is decreased by a factor between 2 and 4 .

\section{Practical Enrollment}

When the quality of enrolled iris images is not perfect, the radius of hyper-dimensional balls needs to be adjusted. In addition to radius $\lfloor\epsilon \times n\rfloor$ bits attributed to the query image quality, we increase the radius of hyper-dimensional balls by the maximum possible number of bits attributed to the reduced quality of enrolled iris classes. If we denote the maximum increment of the radius by $\lfloor\delta \times n\rfloor$, then the recognition threshold is adjusted to $r=\lfloor(\epsilon+\delta) \times n\rfloor$, and the reduced capacity of the IrisCode algorithm applied to reduced quality iris images can be found by applying either Plotkin or EliasBassalygo bound as described in Sections II-B and II-C.

\section{A. Recommendation Regarding Threshold}

Plotkin bound clearly demonstrates that IrisCode does not perform well when the threshold $\epsilon$ is set to 0.32 . Moving the threshold to 0.22 improves the population coverage of IrisCode. According to the Elias-Bassalygo bound, we may be able to recognize up to $1.37 \times 10^{11}$ with a very small possible probability of error. To operate at this threshold, we would have to ensure the highest possible quality of enrolled and even query data. Recall that the threshold 0.22 is the radius of an iris class hyper-dimensional ball composed of the sum of distortions due to enrollment codeword and the query codeword. Therefore, the quality (measured as a radius in units of normalized HD) has to be reduced to $0.11=0.22 / 2$ or below for both enrollment and query iris samples. This level of quality of data is not easy to achieve. However, it is possible.

It is well understood within the scope of biometrics that keeping high quality of biometric data ensures high recognition performance and hence the high population coverage of the recognition system. To improve the quality of iris codes we can apply various signal processing and machine learning approaches. As an example, the quality of iris data may be improved by collecting multiple iris samples per class and then retaining samples characterized by the highest possible quality.

\section{B. Does Performance Scale?}

What does this analysis tell us? Why does IrisCode demonstrate outstanding recognition performance when the threshold is set to 0.32 ? The main caveat lies in the analysis of noise level (the same as the radius of a hyper-dimensional ball representing a single iris class). The typical value of noise in practical data is below 0.25 . As we can see from Table II, for $n=256$ and noise level $\epsilon=0.25$, the iris recognition system can manage 86 iris classes without any error; and 
TABLE III

HAMming (HB), Plotkin (PB), AND EliAs-BASSALYGo (E-BB) BOUNDS FOR CODE LENGTH $n=245$

\begin{tabular}{|c||c|c|c|c|c|c|}
\hline \multicolumn{1}{|c||}{} & \multicolumn{5}{c|}{ Radius in normalized HD } \\
\cline { 2 - 7 } & 0.12 & 0.16 & 0.2 & 0.24 & 0.28 & 0.32 \\
\hline HB & $1.26 \times 10^{36}$ & $1.52 \times 10^{28}$ & $3.82 \times 10^{21}$ & $3.84 \times 10^{16}$ & $8.87 \times 10^{11}$ & $1.52 \times 10^{8}$ \\
\hline PB & & & & & 8 & 4 \\
\hline E-BB & $5.30 \times 10^{34}$ & $2.48 \times 10^{24}$ & $6.98 \times 10^{14}$ & $3.04 \times 10^{6}$ & & \\
\hline
\end{tabular}

TABLE IV

HAMMING AND PLOTKIN BOUNDS FOR CODE LENGTH $n=16$

\begin{tabular}{|c||c|c|c|c|c|c|}
\hline \multicolumn{1}{|c||}{} & \multicolumn{6}{c|}{ Radius in normalized HD } \\
\cline { 2 - 7 } & 0.12 & 0.16 & 0.2 & 0.24 & 0.28 & 0.32 \\
\hline Hamming Bound & 3855 & 478 & 94 & 94 & 26 & 9 \\
\hline Plotkin Bound & & & & & 6 & 2 \\
\hline Actual Capacity & 2048 & 256 & 32 & 32 & 4 & 2 \\
\hline
\end{tabular}

TABLE V

HAMMING AND PLOTKIN BOUNDS FOR CODE LENGTH $n=16$

\begin{tabular}{|c||c|c|c|c|c|c|}
\hline \multicolumn{1}{|c||}{} & \multicolumn{7}{c|}{ Radius in normalized HD } \\
\cline { 2 - 8 } & 0.12 & 0.16 & 0.2 & 0.24 & 0.28 & 0.32 \\
\hline Hamming Bound & 3855 & 478 & 94 & 94 & 26 & 9 \\
\hline Plotkin Bound & & & & & 6 & 2 \\
\hline Actual Capacity & 2048 & 256 & 32 & 32 & 4 & 2 \\
\hline
\end{tabular}

even at radius 0.32 , the overlap between neighboring hyperdimensional balls is small. In addition, this rarely happens under a verification scenario because a query iris is compared to a claimed identity and not to every enrolled class in the iris gallery. This explains the nearly perfect Receiver Operating Curve on small iris datasets, with the population size smaller than 1000 . However, this nearly perfect performance of IrisCode does not scale well to large datasets, especially when a Rank 1 recognition is performed, that is, when a query IrisCode template is compared to enrolled data of every class present in the iris gallery. To achieve a larger population coverage by IrisCode, we have to adjust both quality of enrolled and query data and move the threshold to the left to ensure scalable performance.

\section{Other Binary Codes}

Daugman's IrisCode is not the only algorithm extracting a binary representation from iris images. Other coding approaches are available. For example, the application of 2D median filters followed by comparing filter results from

TABLE VI

ACTUAL POPULATION OF IRISCODE WITH AND WITHOUT \pm 1 BIT CODE ALIGNMENT FOR CODE LENGTH $n=16$

\begin{tabular}{|c||c|c|c|c|c|c|}
\hline \multicolumn{1}{|c||}{} & \multicolumn{7}{c|}{ Radius in normalized HD } \\
\cline { 2 - 7 } & 0.12 & 0.16 & 0.2 & 0.24 & 0.28 & 0.32 \\
\hline No Rotation & 2048 & 256 & 32 & 32 & 4 & 2 \\
\hline \pm 1 bit Rotation & 876 & 63 & 11 & 11 & 4 & 2 \\
\hline
\end{tabular}

different window sizes can also be used to generate a binary code [7]. A comprehensive list of methods that can be used to generate binary code for iris images can be found in the survey paper [8]. As processing steps in all these encoding techniques are different, their tolerance to noise and the number of degrees of freedom are different as well. Despite their encoding difference, the analysis of the maximum population covered by the algorithms can be reduced to the analysis presented in this paper.

\section{Summary}

From the examples and theory presented above, we conclude that the capacity of IrisCode can be analyzed by stating the problem as a basic Rate-Distortion/Channel Coding problem. Within this framework, the task is to find the maximum possible population covered by IrisCode under the constraint on the distance between codewords. They should be separated by more than the minimum normalized HD, which is attributed to noise and distortions present in iris codewords. This model should be used to analyze IrisCode performance of large-scale iris databases, such as those used in National ID programs, where on the one hand the requirement to error rate is excessively stringent and on the other hand the quality of enrolled and query iris data can be controlled.

\section{REFERENCES}

[1] J. Daugman, "Information theory and the iriscode," IEEE Transactions on Information Forensics and Security, vol. 11, no. 2, pp. 400-409, 2016. [Online]. Available: https://doi.org/10.1109/TIFS.2015.2500196

[2] - "How iris recognition works," IEEE Transactions on Circuits and Systems for Video Technology, vol. 14, no. 1, pp. 21-30, Jan 2004.

[3] T. M. Cover and J. A. Thomas, Elements of Information Theory. Hoboken, NJ: Wiley-Interscience, 2006

[4] J. H. Van Lint, "A survey of perfect codes," The Rocky Mountain Journal of Mathematics, vol. 5, no. 2, pp. 199-224, 1975. [Online]. Available: https://www.jstor.org/stable/44236426

[5] M. Plotkin, "Binary codes with specified minimum distance," IRE Transactions on Information Theory, vol. 6, pp. 445-450, 1960.

[6] L. A. Bassalygo, "New upper bounds for error correcting codes," Problems of Information Transmission, vol. 1, pp. 32-35, 1965. [Online]. Available: http://mi.mathnet.ru/ppi762

[7] J. Zuo, N. K. Ratha, and J. H. Connell, "Median filter based iris encoding technique," in 2008 IEEE Second International Conference on Biometrics: Theory, Applications and Systems, 2008, pp. 1-6.

[8] K. W. Bowyer, K. Hollingsworth, and P. J. Flynn, "Image understanding for iris biometrics: A survey," Computer Vision and Image Understanding, vol. 110, no. 2, p. 281-307, May 2008. [Online]. Available: https://doi.org/10.1016/j.cviu.2007.08.005 